\title{
THE FORMULATION OF SECOND-ORDER BOUNDARY VALUE PROBLEMS ON TIME SCALES
}

\author{
FORDYCE A. DAVIDSON AND BRYAN P. RYNNE
}

Received 6 February 2006; Revised 21 April 2006; Accepted 15 May 2006

We reconsider the basic formulation of second-order, two-point, Sturm-Liouville-type boundary value problems on time scales. Although this topic has received extensive attention in recent years, we present some simple examples which show that there are certain difficulties with the formulation of the problem as usually used in the literature. These difficulties can be avoided by some additional conditions on the structure of the time scale, but we show that these conditions are unnecessary, since in fact, a simple, amended formulation of the problem avoids the difficulties.

Copyright (c) 2006 F. A. Davidson and B. P. Rynne. This is an open access article distributed under the Creative Commons Attribution License, which permits unrestricted use, distribution, and reproduction in any medium, provided the original work is properly cited.

\section{Introduction}

In the time scale literature, there has been considerable interest in Sturm-Liouville boundary value problems of the form

$$
-\left[p u^{\Delta}\right]^{\Delta}(t)+q(t) u^{\sigma}(t)=f\left(\lambda, t, u^{\sigma}(t)\right), \quad[a, b] \cap \mathbb{T}^{\kappa^{2}}
$$

for suitable functions $p, q$, and $f$ and real parameter $\lambda$, together with boundary conditions, which are generally taken to have the form

$$
\begin{gathered}
\alpha u(a)+\beta u^{\Delta}(a)=0, \\
\gamma u\left(\sigma^{2}(b)\right)+\delta u^{\Delta}(\sigma(b))=0,
\end{gathered}
$$

for some points $a, b \in \mathbb{T}$, with $a<b$ and $(|\alpha|+|\beta|)(|\gamma|+|\delta|)>0$ (see, e.g., $[1,2,6,7$, $9,11,15]$ and in the case of systems [12]) although there are other formulations of the boundary conditions (see Remark 3.1). The formulation (1.1)-(1.2) covers both linear eigenvalue problems and nonlinear problems. 
At first sight, (1.1)-(1.2) seems to be a reasonable formulation of a boundary value problem and, indeed, it has received considerable attention. However, in this paper, we show that for general time scales, there are certain difficulties with this basic formulation. These difficulties can be avoided by some additional conditions on the structure of the time scale, but we show that these conditions are unnecessary, since in fact a simple, amended formulation of the problem avoids the difficulties.

The difficulties just mentioned concern the basic formulation of the linear operator formed from the left-hand side of (1.1), together with the boundary conditions (1.2), so from now on we simply consider the formulation of this linear operator and ignore the right-hand side of (1.1). In Section 2, we briefly recall some basic definitions and results concerning time scales-further details can be found in, for example, $[4,8,14,16]$. In Section 3, we then describe the standard boundary value formulation more precisely, and give some simple examples to highlight various problems with this formulation. A formulation that avoids these problems is then presented in Section 4. In Section 5, we consider the closely related $\Delta \nabla$-problem.

\section{Preliminaries}

A time scale $\mathbb{T}$ is a nonempty, closed subset of $\mathbb{R}$. We will restrict our attention to bounded time scales. We endow $\mathbb{T}$ with the subspace topology inherited from $\mathbb{R}$.

Let

$$
i_{\mathbb{T}}:=\inf \{s \in \mathbb{T}\}, \quad s_{\mathbb{T}}:=\sup \{s \in \mathbb{T}\}
$$

Define the jump operators $\sigma, \rho: \mathbb{T} \rightarrow \mathbb{T}$ by

$$
\sigma(t):=\inf \{s \in \mathbb{T}: s>t\}, \quad \rho(t):=\sup \{s \in \mathbb{T}: s<t\}, \quad t \in \mathbb{T},
$$

where, in this definition, we write inf $\varnothing=i_{\mathbb{T}}$, $\sup \varnothing=s_{\mathbb{T}}$, so that $\rho\left(i_{\mathbb{T}}\right)=i_{\mathbb{T}}$ and $\sigma\left(s_{\mathbb{T}}\right)=s_{\mathbb{T}}$. A point $t \in \mathbb{T}$ is said to be left-dense, left-scattered, right-dense, right-scattered if $\rho(t)=t$, $\rho(t)<t, \sigma(t)=t, \sigma(t)>t$, respectively.

Now suppose that $u: \mathbb{T} \rightarrow \mathbb{R}$. Continuity of $u$ is defined in the usual manner, while $u$ is said to be $r d$-continuous on $\mathbb{T}$ if it is continuous at all right-dense points in $\mathbb{T}$ and has finite-left-sided limits at all left-dense points. We let $C_{\mathrm{rd}}^{0}(\mathbb{T})\left(\right.$ resp., $C^{0}(\mathbb{T})$ ) denote the set of rd-continuous (resp., continuous) functions $u: \mathbb{T} \rightarrow \mathbb{R}$, and let

$$
|u|_{0, \mathbb{\mathbb { T }}}:=\sup \{|u(t)|: t \in \mathbb{T}\}, \quad u \in C_{\mathrm{rd}}^{0}(\mathbb{T}) .
$$

With this norm, these spaces are Banach spaces. For any $u$, we let $u^{\sigma}:=u \circ \sigma$; if $u \in C^{0}(\mathbb{T})$ then $u^{\sigma} \in C_{\text {rd }}^{0}(\mathbb{T})$.

We assume throughout that $\rho\left(s_{\mathbb{T}}\right)>i_{\mathbb{T}}$, so that $\mathbb{T}$ must contain at least 3 points. Now define the set

$$
\mathbb{T}^{\mathcal{\kappa}}:= \begin{cases}\mathbb{T} \backslash\left\{s_{\mathbb{T}}\right\} & \text { if } \rho\left(s_{\mathbb{T}}\right)<s_{\mathbb{T}}, \\ \mathbb{T} & \text { otherwise. }\end{cases}
$$


The set $\mathbb{T}^{\kappa}$ is closed (its construction simply removes the maximal point from $\mathbb{T}$ if this point is isolated, which leaves a closed set), so $\mathbb{T}^{\kappa}$ is a time scale, and hence all the above constructions can be applied to $\mathbb{T}^{\kappa}$. In particular, we can define the above Banach spaces and norms using $\mathbb{T}^{\kappa}$ instead of $\mathbb{T}$.

A function $u: \mathbb{T} \rightarrow \mathbb{R}$ is differentiable at $t \in \mathbb{T}^{\kappa}$ if there exists a number $u^{\Delta}(t)$ with the following property: for any $\epsilon>0$, there exists $\delta>0$ such that

$$
s \in \mathbb{T}, \quad|t-s|<\delta \Longrightarrow\left|u(\sigma(t))-u(s)-u^{\Delta}(t)(\sigma(t)-s)\right| \leq \epsilon|\sigma(t)-s| .
$$

We note that if $s_{\mathbb{U}}$ is left-scattered, then this would not define $u^{\Delta}\left(s_{\mathbb{U}}\right)$; however, this is exactly the case for which $s_{\mathbb{T}} \notin \mathbb{T}^{\kappa}$, and we do not attempt to define $u^{\Delta}\left(s_{\mathbb{T}}\right)$ in this case.

If $u$ is differentiable at every $t \in \mathbb{T}^{\kappa}$, then $u$ is said to be $(\Delta-)$ differentiable. It can be shown that if $u$ is differentiable at $t$, then $u$ is continuous at $t$, and so, if $u$ is differentiable, then $u \in C^{0}(\mathbb{T})$. Let $C_{\mathrm{rd}}^{1}(\mathbb{T})$ (resp., $\left.C^{1}(\mathbb{T})\right)$ denote the set of functions $u \in C^{0}(\mathbb{T})$ which are differentiable and for which $u^{\Delta} \in C_{\text {rd }}^{0}\left(\mathbb{T}^{\mathcal{K}}\right)$ (resp., $u^{\Delta} \in C^{0}\left(\mathbb{T}^{\mathcal{K}}\right)$ ). With the norm

$$
|u|_{1, \mathbb{I}}:=|u|_{0, \mathbb{\Psi}}+\left|u^{\Delta}\right|_{0, \mathbb{}}, \quad u \in C_{\mathrm{rd}}^{1}(\mathbb{T}),
$$

these spaces are again Banach spaces.

To define the second derivative of $u$, we define the time scale $\mathbb{T}^{\kappa^{2}}:=\left(\mathbb{T}^{\kappa}\right)^{\kappa}$, and then the second derivative of $u$ at $t \in \mathbb{T}^{\kappa^{2}}$ is defined to be $u^{\Delta \Delta}(t):=\left(u^{\Delta}\right)^{\Delta}(t)$. We can also define Banach spaces $C_{\mathrm{rd}}^{2}(\mathbb{T}), C^{2}(\mathbb{T})$ in a similar manner to the above definitions of $C_{\mathrm{rd}}^{1}(\mathbb{T})$, $C^{1}(\mathbb{T})$, with the norm

$$
|u|_{2, \mathbb{\mathbb { T }}}:=|u|_{0, \mathbb{\mathbb { T }}}+\left|u^{\Delta}\right|_{0, \mathbb{\mathbb { T }}^{\kappa}}+\left|u^{\Delta \Delta}\right|_{0, \mathbb{\mathbb { N }}^{2}}, \quad u \in C_{\mathrm{rd}}^{2}(\mathbb{\mathbb { T }}) .
$$

Remark 2.1. The definition of the Banach spaces given above is not a point of pedantry. A correct definition of a function space (with correctly defined norm) in which to search for a solution of a problem is essential for any meaningful discussion of the problem. Of course, alternatives to the spaces described above could be utilised. Unfortunately, many papers in the existing time scale literature omit any precise definition of the space they are using, or at best give only a partial definition, despite a correct definition of the basic Banach space being fundamental to the analytic techniques employed (e.g., fixed point theorems, in e.g., $[6,7,10,12,15])$. For example, the papers $[10,15]$ define the space $E:=\{u:[a, \sigma(b)] \rightarrow \mathbb{R}\}$, with the norm $\|u\|:=\sup _{a \leq t \leq \sigma(b)}|u(t)|$. No continuity, or even measurability, conditions are imposed on the functions in $E$, despite the fact that the ability to integrate the functions in $E$ is central to the proofs contained in these papers. As defined, $E$ is certainly a Banach space, but the elements of $E$ need not even be measurable (supposing the time scale to be a real interval, on which we have a well-defined idea of measurability), let alone integrable. In fact, these papers use the standard idea of integrability on time scales in terms of antiderivatives, which imposes more stringent conditions than simply measurability, so it is clear that further conditions are required to yield a suitable Banach space. 


\section{The standard formulation of a linear operator}

A basic starting point to most discussions of boundary value problems is the construction of a linear operator, on suitable function spaces, which represents the differential operator part of the problem, that is, the left-hand side of (1.1), and incorporates the boundary conditions (1.2). We now describe the standard formulation of such a linear operator, and then discuss some problems with this formulation.

Let $\mathbb{T}$ be a bounded time scale, and $a, b \in \mathbb{T}$, with $a<b$. For a real-valued function $u$ satisfying the boundary conditions (1.2) define

$$
\widetilde{L} u(t):=-\left[p(t) u^{\Delta}\right]^{\Delta}(t)+q(t) u^{\sigma}(t), \quad t \in[a, b] \cap \mathbb{T}^{\kappa^{2}}
$$

for suitable functions $p, q$ and suitable $u$. Clearly, some continuity and differentiability conditions need to be imposed on the functions $p$ and $q$ but this is not our main interest at the present moment, so we postpone this until the next section, and consider the conditions on $u$. In addition, it is assumed that $u$ satisfies the boundary conditions in (1.2). These conditions are imposed in, for example, $[1,6,9,11]$. A common alternative form for the boundary conditions is described in the following remark.

Remark 3.1. In almost all the existing literature, the left-hand boundary condition has the form stated in (1.2), but the right-hand boundary condition has often been expressed in the alternative form

$$
\tilde{\gamma} u(\sigma(b))+\tilde{\delta} u^{\Delta}(\sigma(b))=0
$$

(see, e.g., $[7,10,15])$. Clearly, if $\sigma(b)=\sigma^{2}(b)$, there is no distinction between these two formulations. However, even if $\sigma^{2}(b)>\sigma(b)$, these two formulations are in fact equivalent (albeit, possibly with different values of the coefficients $\gamma, \delta$ and $\tilde{\gamma}, \tilde{\delta}$ ) since the definition of the derivative can be used to rewrite each condition in the form

$$
c_{1} u(\sigma(b))+c_{2} u\left(\sigma^{2}(b)\right)=0
$$

for a unique (up to rescaling) pair of coefficients $\left(c_{1}, c_{2}\right) \in \mathbb{R}^{2}$, where $\left(c_{1}, c_{2}\right)$ is independent of whether we start with (1.2) or (3.2).

It should be noted, however, that the form adopted in (3.2) has a slight advantage in certain calculations which involve taking the inner product of $\tilde{L} u$ with $u^{\sigma}$ and integrating by parts. Furthermore, in this case, it is often appropriate to suppose that $\tilde{\gamma}, \tilde{\delta} \geq 0$, which is not equivalent to $\gamma, \delta \geq 0$.

We note that even at this stage in the formulation of $\widetilde{L}$, many papers in the literature are rather vague about the domains of both the function $u$ and the operator $\tilde{L}-$ in fact, often no mention is made at all of what domain either $u$ or $\widetilde{L}$ is defined on. In Section 4 , we will give an alternative formulation in which the domains of $u$ and $\widetilde{L}$ will be precisely defined, but for now, we continue with the discussion of the above standard formulation. 
In order for the derivative $u^{\Delta}(\sigma(b))$ in the boundary condition (1.2) to be defined, the domain of $u$ needs to include the set $([a, b] \cap \mathbb{T}) \cup\left\{\sigma(b), \sigma^{2}(b)\right\}$, but presumably is not taken to be all of $\mathbb{T}$ if $\mathbb{T}$ is considerably bigger than this set. Unfortunately, many papers fail to make clear what the relationship between the points $\left\{a, b, \sigma(b), \sigma^{2}(b)\right\}$ and the sets $\mathbb{T}, \mathbb{T}^{\kappa}, \mathbb{T}^{\kappa^{2}}$ is. In fact, $a$ and $b$ usually appear to be regarded as arbitrary points in $\mathbb{T}$.

In addition, in order for the second derivative term in (3.1) to be defined, it is necessary that the domain of $\tilde{L} u$ in $(3.1)$ be contained in $\mathbb{T}^{\kappa^{2}}$ (see Section 2), so the domain $[a, b] \cap \mathbb{T}^{\kappa^{2}}$ stated in (3.1) is reasonable. Some papers do not state any domain for $\tilde{L} u$, however, it is clear that, in fact, most papers take the domain of $\tilde{L} u$ in $(3.1)$ to be the set $[a, b] \cap \mathbb{T}$ (explicitly or implicitly), see for example $[1,7,10,11]$. Clearly, for this to make sense, it is necessary to impose the condition that

$$
b \in \mathbb{T}^{\kappa^{2}} .
$$

This condition is usually not stated explicitly, but is necessary whenever $b$ is taken to be in the domain of $\tilde{L} u$. In addition, in order for the second boundary condition in (1.2) to make sense, it is also necessary to impose the condition that

$$
\sigma(b) \in \mathbb{T}^{\kappa} .
$$

As mentioned above, most papers on time scale boundary value problems use the above formulation, but fail to impose any condition on the relationship between the points $\left\{b, \sigma(b), \sigma^{2}(b)\right\}$ and the sets $\mathbb{T}, \mathbb{T}^{\kappa}, \mathbb{T}^{\kappa^{2}}$, despite the necessity of the conditions (3.4) and (3.5). The following example gives a concrete illustration of the above remarks and shows that the conditions (3.4) and (3.5) are actually different, and that they can fail to hold.

Example 3.2. Let

$$
\begin{aligned}
& \mathbb{T}_{1}=[-1,0] \cup\{1\}, \\
& \mathbb{T}_{2}=[-1,0] \cup\{1,2\} .
\end{aligned}
$$

Clearly,

$$
\begin{gathered}
\mathbb{T}_{1}^{\mathcal{K}}=\mathbb{T}_{1}^{\kappa^{2}}=[-1,0], \quad \mathbb{T}_{2}^{\kappa}=[-1,0] \cup\{1\}, \\
\mathbb{T}_{2}^{\mathcal{K}^{2}}=[-1,0] .
\end{gathered}
$$

We suppose that $a=-1$ in each case. If we now choose $b=1$, then $\mathbb{T}_{1}$ and $\mathbb{T}_{2}$ fail to satisfy either (3.4) or (3.5). On the other hand, if we choose $b=0$, then $\mathbb{T}_{1}$ satisfies (3.4) but not (3.5), while $\mathbb{T}_{2}$ satisfies both conditions.

Remark 3.3. Perhaps as an alternative to condition (3.4), the condition

$$
\sigma^{2}(b) \in \mathbb{T}
$$

has been used (see, e.g., [9]). However, given that the function $\sigma$ is defined as a mapping from $\mathbb{T}$ to $\mathbb{T}$, it seems to be impossible to have $\sigma^{2}(b) \notin \mathbb{T}$. Some papers also omit the 
definitions of $\sigma\left(s_{\mathbb{}}\right)$ and $\rho\left(i_{\mathbb{}}\right)$ (see, e.g., $\left.[1,9,11,13,15]\right)$, but this does not appear to help ensure that (3.4) holds.

Even if both the conditions (3.4) and (3.5) hold, there is still a problem with the above formulation. Consider the time scale $\mathbb{T}_{3}=[-1,0] \cup[1,3]$, with $a=-1, b=1$, so that $[a, b] \cap \mathbb{T}_{3}=\mathbb{T}_{1}\left(\mathbb{T}_{1}\right.$ as in Example 3.2). Clearly, (3.4) and (3.5) hold; in fact, the point $b$ is far removed from $s_{\mathbb{T}_{3}}$, so these conditions are irrelevant here. However, the above formulation still has problems. As it stands, the boundary condition (1.2) requires the derivative $u^{\Delta}(1)$ to be evaluated which, by the definition of the derivative $u^{\Delta}$ in Section 2, requires $u$ to be defined on some (arbitrarily small) interval to the right of 1 (admittedly, there has been some recent discussion of "boundary value" problems with the "boundary value" prescribed in the interior of the interval, but this does not seem to be what was intended in the literature to which we are referring). However, the function $\tilde{L} u$ is not defined to the right of 1 , and it does not seem that a boundary value problem of this form will provide values of $u$ to the right of 1 . The problem here is caused by formulating the problem firstly in terms of the time scale $\mathbb{T}_{3}$, which is then restricted to the time scale $\mathbb{T}_{1}$, but the necessary conditions (3.4) and (3.5) are stated in terms the original time scale $\mathbb{T}_{3}$ and $\mathbb{T}_{3}^{\kappa}, \mathbb{T}_{3}^{\kappa^{2}}$. In this case, the set $(1,3] \subset \mathbb{T}_{3}$ is simply redundant and merely serves to confuse matters.

The solution to this dilemma is simply to recognise that if one desires to consider a boundary value problem on a closed subset $\tilde{T}$ of a time scale $\mathbb{T}$, then $\tilde{T}$ should be taken to be the basic time scale for the problem, and the remainder of the original time scale $\mathbb{T}$ discarded. Moreover, the boundary values should be set up in terms of the end points of $\widetilde{T}$, with any derivatives being defined at points appropriately "stepped in" from these end points - in fact, we describe such a formulation in Section 4.

Most of the difficulties discussed above occur when it happens that $\sigma(b)=\sigma^{2}(b)$, so these difficulties could be avoided simply by restricting attention to problems for which $\sigma(b)<\sigma^{2}(b)$. However, this excludes an entire class of problems which could easily be included by using an alternative formulation of the boundary value problem, as will be discussed in Section 4.

Remark 3.4. It is not only the setup that suffers under the above formulation, it also leads to mistaken arguments. For example, in the proof of [6, Lemma 3.1], it is stated that if $\sigma^{2}(1)$ is left-scattered, then the solution $x$ constructed there has $x(\sigma(1))>0$. However, this statement is incorrect on any time scale with $\sigma(1)=\sigma^{2}(1)$ (e.g., $\mathbb{T}_{1}$ above), since then $x(\sigma(1))=x\left(\sigma^{2}(1)\right)=0$ (under the hypotheses in [6]).

\section{A consistent formulation}

In this section, we describe a consistent formulation of a linear operator with which to describe the two-point boundary value problems of interest here.

Let $\mathbb{T}$ be a bounded time scale with $a=i_{\mathbb{T}}$ and $b=s_{\mathbb{T}}$. Suppose that $p \in C^{1}\left(\mathbb{T}^{\kappa}\right)$, $q \in C_{\mathrm{rd}}^{0}\left(\mathbb{T}^{\kappa^{2}}\right)$, and $p \geq c>0$, on $\mathbb{T}^{\kappa}$, for some constant $c>0$. We define the boundary conditions in the form

$$
\alpha u(a)+\beta u^{\Delta}(a)=0, \quad \gamma u(b)+\delta u^{\Delta}(\rho(b))=0
$$


with $(|\alpha|+|\beta|)(|\gamma|+|\delta|)>0$. (As with the standard formulation of the boundary conditions, we could use the value $u(b)$ or $u(\rho(b))$ in (4.1), since by similar arguments to those in Remark 3.1, there is no essential difference between these alternative formulations.) Define $L: D(L) \subset C_{\mathrm{rd}}^{2}(\mathbb{T}) \rightarrow C_{\mathrm{rd}}^{0}\left(\mathbb{T}^{2}\right)$ by

$$
\begin{gathered}
D(L):=\left\{u \in C_{\mathrm{rd}}^{2}(\mathbb{T}): u \text { satisfies }(4.1)\right\}, \\
L u(t):=-\left[p u^{\Delta}\right]^{\Delta}(t)+q(t) u^{\sigma}(t), \quad t \in \mathbb{T}^{\kappa^{2}}, u \in D(L) .
\end{gathered}
$$

This definition of the linear operator $L$ is unambiguous about domains, and ensures that irrespective of the structure of the time scale $\mathbb{T}$, all functions and derivatives in the boundary conditions and the operator are only evaluated at points where they are well defined. In particular, the first derivatives (in the operator and in the boundary condition) and the second derivative (in the operator) are only evaluated on the correct subsets of $\mathbb{T}$, namely $\mathbb{T}^{\kappa}$ and $\mathbb{T}^{\kappa^{2}}$, respectively.

The formulation proposed here appears only slightly different from the formulation previously described. However, key differences between these formulations are

(a) the domains of $u$ and $L$ are explicitly defined - in particular, there is no ambiguity about which portions of $\mathbb{T}$ are of interest (all of $\mathbb{T}$ is relevant);

(b) the first derivatives in the boundary conditions (4.1) and in the expression for $L u$ and the second derivative in the expression for $L u$ are only evaluated on $\mathbb{T}^{\mathcal{k}}$ and $\mathbb{T} \kappa^{2}$, respectively, that is, where these derivatives make sense;

(c) none of the difficulties discussed in the previous section arise for this formulation.

The main difference between the formulations in this section and in the previous section (apart from precision in domains) lies in the points at which to define the boundary conditions. In (1.2), the right-hand boundary condition is expressed in terms of $\sigma(b)$ and $\sigma^{2}(b)$, that is, by moving upwards from $b$ or "outwards" from $\mathbb{T}$, whereas in (4.1), the right-hand boundary condition is expressed in terms of $b$ and $\rho(b)$, that is, by moving "inwards" into $\mathbb{T}$. This formulation avoids all the difficulties discussed previously. Of course, as remarked at the end of Section 3, these difficulties could be avoided simply by restricting the class of time scales considered, but this unnecessarily excludes an entire class of time scales which could easily be included by using the above formulation.

\section{5. $\nabla$-derivative operators}

In addition to the "forward difference" $\Delta$-derivative considered above, a "backward difference" $\nabla$-derivative can be defined in a similar manner (see [4]), and hence boundary value operators containing $\nabla$-derivatives can be defined. We consider this very briefly here.

First, define the time scale $\mathbb{T}_{\kappa}$ (analogous to $\mathbb{T}^{\kappa}$ ) by removing the minimal point from $\mathbb{T}$ if this point is isolated. Now suppose that $u: \mathbb{T} \rightarrow \mathbb{R}$. The function $u$ is called ld-continuous if it is continuous at all left-dense points and has finite-right sided limits at all rightdense points. Then a "backward difference" derivative $u^{\nabla}: \mathbb{T}_{\kappa} \rightarrow \mathbb{R}$ can be defined in a similar manner to $u^{\Delta}$. A parallel construction to the above then yields Banach spaces 
$C_{\text {ld }}^{0}(\mathbb{T}), C_{\mathrm{ld}}^{\nabla}(\mathbb{T}), C^{0}(\mathbb{T})$, and $C^{\nabla}(\mathbb{T})$, with the natural sup norms. The second-order derivative $u^{\nabla \nabla}: \mathbb{T}_{\mathcal{K}^{2}} \rightarrow \mathbb{R}$ and two mixed second-order derivatives $u^{\Delta \nabla}, u^{\nabla \Delta}: \mathbb{T}_{\kappa}^{\kappa} \rightarrow \mathbb{R}$ can then be defined in a natural manner, with associated Banach spaces of twice differentiable functions. Clearly, a variety of second-order differential operators can then be constructed, with appropriate boundary conditions. Most of the considerations regarding suitable domains and points at which to impose the boundary conditions discussed above also apply to these alternative operators.

We illustrate one construction. Suppose that $p \in C^{\nabla}\left(\mathbb{T}_{\kappa}\right), q \in C^{0}\left(\mathbb{T}_{\kappa}^{\kappa}\right)$, and $p \geq c>0$, on $\mathbb{T}_{\kappa}$, for some constant $c>0$. Consider the boundary conditions

$$
\alpha u(a)+\beta u^{\Delta}(a)=0, \quad \gamma u(b)+\delta u^{\Delta}(\rho(b))=0,
$$

with $(|\alpha|+|\beta|)(|\gamma|+|\delta|)>0$, and define $K: D(K) \subset C^{\Delta \nabla}(\mathbb{T}) \rightarrow C^{0}\left(\mathbb{T}_{\kappa}^{\kappa}\right)$ by

$$
\begin{gathered}
D(K):=\left\{u \in C^{\Delta \nabla}(\mathbb{T}): u \text { satisfies }(5.1)\right\}, \\
K u:=-\left[p u^{\Delta}\right]^{\nabla}(t)+q(t) u(t), \quad t \in \mathbb{T}_{\kappa}^{\kappa}, u \in D(K) .
\end{gathered}
$$

Remark 5.1. The above $\Delta \nabla$ formulation (or a similar $\nabla \Delta$ formulation) has the advantage of making the second derivative operator in $K$ "centred," and hence makes it appropriate to replace the rd-continuous term $u^{\sigma}$ in $L$ with the continuous term $u$ in $K$. This has the advantage that the solution $u$ of an equation of the form $K u=h$ with $h \in C^{0}(\mathbb{T})$ will be twice continuously differentiable, that is, $u \in C^{\Delta \nabla}(\mathbb{T})$ (rather than $u \in C_{\mathrm{rd}}^{2}(\mathbb{T})$, which would be the case with an analogous $L$ equation). This consideration motivates the domain and range of $K$ chosen above.

Again, the above formulation of $K$ is different to that typically used in the previous literature (see, e.g., $[3,17,18]$ ), but is again motivated by the desire to produce a precise and unambiguous definition of $K$, and to ensure that derivatives are only evaluated at points at which they are defined, for all classes of time scales (we observe that in, e.g., $[3,18]$, the problem of evaluating derivatives at points outwith their normal domain of definition can again occur for certain time scales).

Remark 5.2. Another motivation for studying the operator $K$, as opposed to the operator $L$ considered in Section 4, is to obtain a selfadjoint operator. Standard linear functional analysis shows that selfadjoint operators on Hilbert spaces have a host of desirable properties not possessed by general, non-selfadjoint operators. Of course, even in the real setting, in order to obtain selfadjoint operators, it is necessary to use $L^{2}$-type Hilbert spaces rather than Banach spaces of continuous functions. However, many of the desirable properties of selfadjoint operators can be obtained, even on such Banach spaces, if the Banach space operator is symmetric with respect to a suitable $L^{2}$-type inner product, and possesses a selfadjoint extension to a larger $L^{2}$-type Hilbert space-in this context the term "formally selfadjoint" is sometimes used for the underlying Banach space operator.

Of course, to date, most time scales papers use Banach spaces of continuous (or rd-or ld-continuous functions) rather than Hilbert spaces of $L^{2}$-type functions. Despite this, 
the operator $L$ defined in Section 4 has often been termed "selfadjoint" (see, e.g., [4, Chapter 4] and the references therein). This would not be unreasonable if $L$ at least possessed a selfadjoint extension. However, it was shown in [8, Section 6] that $L$ cannot possibly have any such selfadjoint extension (at least, on any Hilbert space containing the above domain $D(L)$ ). Thus this terminology seems inappropriate for $L$. However, the operator $K$ is symmetric with respect to an $L^{2}$ inner product (see [5, Chapter 4] and the references therein), at least with appropriate boundary conditions. Thus, it seems that the term "selfadjoint" may be appropriate in this setting-this remains to be proven and requires the full development of an $L^{2}$ theory in the general time scale setting.

\section{References}

[1] R. P. Agarwal and D. O'Regan, Nonlinear boundary value problems on time scales, Nonlinear Analysis 44 (2001), no. 4, 527-535.

[2] P. Amster, C. Rogers, and C. C. Tisdell, Existence of solutions to boundary value problems for dynamic systems on time scales, Journal of Mathematical Analysis and Applications 308 (2005), no. 2, 565-577.

[3] F. M. Atici and G. Sh. Guseinov, On Green's functions and positive solutions for boundary value problems on time scales, Journal of Computational and Applied Mathematics 141 (2002), no. 1-2, 75-99.

[4] M. Bohner and A. Peterson, Dynamic Equations on Time Scales, Birkhäuser, Massachusetts, 2001.

[5] M. Bohner and A. Peterson (eds.), Advances in Dynamic Equations on Time Scales, Birkhäuser, Massachusetts, 2003.

[6] C. J. Chyan, J. M. Davis, J. Henderson, and W. K. C. Yin, Eigenvalue comparisons for differential equations on a measure chain, Electronic Journal of Differential Equations 1998 (1998), no. 35, $1-7$.

[7] C. J. Chyan and J. Henderson, Eigenvalue problems for nonlinear differential equations on a measure chain, Journal of Mathematical Analysis and Applications 245 (2000), no. 2, 547-559.

[8] F. A. Davidson and B. P. Rynne, Global bifurcation on time scales, Journal of Mathematical Analysis and Applications 267 (2002), no. 1, 345-360.

[9] L. Erbe and A. Peterson, Eigenvalue conditions and positive solutions, Journal of Difference Equations and Applications 6 (2000), no. 2, 165-191.

[10]_, Positive solutions for a nonlinear differential equation on a measure chain, Mathematical and Computer Modelling 32 (2000), no. 5-6, 571-585.

[11] L. Erbe, A. Peterson, and R. Mathsen, Existence, multiplicity, and nonexistence of positive solutions to a differential equation on a measure chain, Journal of Computational and Applied Mathematics 113 (2000), no. 1-2, 365-380.

[12] J. Henderson, A. Peterson, and C. C. Tisdell, On the existence and uniqueness of solutions to boundary value problems on time scales, Advances in Difference Equations 2004 (2004), no. 2, 93-109.

[13] J. Henderson and C. C. Tisdell, Topological transversality and boundary value problems on time scales, Journal of Mathematical Analysis and Applications 289 (2004), no. 1, 110-125.

[14] S. Hilger, Analysis on measure chains-a unified approach to continuous and discrete calculus, Results in Mathematics 18 (1990), no. 1-2, 18-56.

[15] C.-H. Hong and C.-C. Yeh, Positive solutions for eigenvalue problems on a measure chain, Nonlinear Analysis 51 (2002), no. 3, 499-507.

[16] V. Lakshmikantham, S. Sivasundaram, and B. Kaymakcalan, Dynamic Systems on Measure Chains, Mathematics and Its Applications, vol. 370, Kluwer Academic, Dordrecht, 1996. 


\section{Boundary value problems}

[17] H.-R. Sun and W.-T. Li, Positive solutions for nonlinear three-point boundary value problems on time scales, Journal of Mathematical Analysis and Applications 299 (2004), no. 2, 508-524.

[18] S. G. Topal, Second-order periodic boundary value problems on time scales, Computers \& Mathematics with Applications 48 (2004), no. 3-4, 637-648.

Fordyce A. Davidson: Division of Mathematics, University of Dundee, Dundee DD1 4HN,

Scotland, UK

E-mail address: fdavidso@maths.dundee.ac.uk

Bryan P. Rynne: Department of Mathematics, Heriot-Watt University, Edinburgh EH14 4AS, Scotland, UK

E-mail address: bryan@ma.hw.ac.uk 